\title{
Elaboración de algoritmos para el diagnóstico diferencial de enfermedades digestivas y respiratorias del cerdo
}

\section{Use of algorithms for the differential diagnosis of digestive and respiratory infectious disorders of pigs}

\author{
Alfonso Carbonero-Martínez*, Raquel Sánchez-Céspedes, Ignacio García- \\ Bocanegra, Antonio Arenas-Casas, Inmaculada Luque-Moreno, Carmen \\ Tarradas-Iglesias, David Cano-Terriza \& Carmen Borge-Rodríguez
}

Fecha de recepción: 09/09/2019; Fecha de revisión: 21/10/2019; Fecha de aceptación: 27/11/2019

Cómo citar este artículo:

Carbonero, A., Sánchez-Céspedes, R., García-Bocanegra, I., Arenas, A., Luque, I., Tarradas, C., Cano, D. \& Borge, C. (2019). Elaboración de algoritmos para el diagnóstico diferencial de enfermedades digestivas y respiratorias del cerdo. Revista de Innovación y Buenas Prácticas Docentes, 8(4),16-23.

Autor de Correspondencia: espargen@hotmail.com

\begin{abstract}
Resumen:
La utilidad de los algoritmos en las especialidades médicas ha sido ampliamente documentada, aunque su empleo en sanidad animal resulta poco frecuente. Para este estudio se ha creado un algoritmo que incluyó el diagnóstico diferencial de los procesos infecciosos respiratorios y digestivos en la especie porcina. A continuación, se realizó una práctica con alumnos de tercer curso del Grado en Veterinaria de la asignatura "Enfermedades Infecciosas", a los que se les ha facilitado información y la posibilidad de solicitar hasta cuatro pruebas diagnósticas laboratoriales para identificar correctamente el agente etiológico. Tras un período para la resolución del caso, en la que solo algunos grupos identificaron correctamente la enfermedad, se les facilitó el algoritmo, lo que permitió que todos los alumnos acertaran el diagnóstico. Finalizada la práctica, a los alumnos se les pasó una encuesta acerca de su experiencia con los algoritmos y su grado de satisfacción. El uso de algoritmos ha demostrado ser útil para la resolución de casos en la asignatura de enfermedades infecciosas según el $100 \%$ de los alumnos, habiendo un $96,9 \%$ de los mismos que los consideran útiles en el ámbito profesional. La práctica ha sido evaluada con un elevado grado de satisfacción (8,72 de media).
\end{abstract}

Palabras clave: algoritmos, diagnóstico, casos clínicos.

\begin{abstract}
:
The use of algorithms in medical specialties has been well documented. However, its use in animal health is not frequent. For this study, an algorithm on differential diagnosis of porcine infectious digestive and respiratory disorders has been designed. Then, a practice was carried out with third year students of the Degree in Veterinary Science enrolled in the subject "Infectious Diseases". During this practice, information on the outbreak was provided to the students, which had the possibility to request until four laboratory tests in order to identify the etiological agent. After a time for the resolution of the case, in which only some groups determined correctly the cause of the outbreak, the algorithm was provided to the students, which allowed to all of them the correct diagnosis of the disorder. After the practice, the students made a survey about their experience with the algorithms and their satisfaction degree. The use of algorithms was useful for the resolution of cases in the subject of infectious diseases according to $100 \%$ of the students, with $96.9 \%$ of them considering them useful in the professional work. The practice has been evaluated with a high degree of satisfaction (8.72 on average).
\end{abstract}

Key Words: algorithms, diagnose, clinical cases. 


\section{INTRODUCCIÓN}

El diagnóstico de distintas patologías supone una de las actividades fundamentales en la práctica veterinaria en casi todos los ámbitos, siendo, a su vez, la base de la lucha frente a las enfermedades, que es una de las competencias que ha de adquirir todo alumno y el fin último de la profesión veterinaria desde un punto de vista clínico y sanitario

La metodología docente imperante en las distintas asignaturas relacionadas con el diagnóstico consiste en impartir las enfermedades como entes individuales con todas sus características, incluidas la etiología, epidemiología, clínica, lesiones, diagnóstico laboratorial y lucha. Sin embargo, cuando el profesional veterinario se enfrenta a un caso, se encuentra, en primer lugar, un conjunto de signos clínicos y lesiones, debiendo recorrer el camino inverso para determinar finalmente cuál es la enfermedad implicada en el proceso. Deberá entonces repasar mentalmente todas las enfermedades estudiadas, compartimentalizadas en distintas asignaturas, buscando compatibilidades con los signos observados y realizar un complejo proceso mediante el que llegar a la conclusión de un diagnóstico correcto. Para ayudar a este fin, en medicina humana se emplean desde hace un tiempo considerable los algoritmos (Chandramohan, Jaffar \& Greenwood, 2002, p. 45; Martin, Doig, Heyland, Morrison, \& Sibbald, 2004, p. 197; Di Domenicantonio et al., 2019, p. 88), claves en su mayoría dicotómicas que permiten enfrentarse a los distintos síndromes clínicos de una manera reglada y sistematizada. Un algoritmo se define como un diagrama orientador que sirve para tomar decisiones diagnósticas o terapéuticas. En este sentido, se parte de un conjunto de signos clínicos (síndrome) y lesiones, avanzándose por la clave hasta identificar la causa del proceso, sea cuál sea la etiología. Un algoritmo sería como un árbol que se va ramificando continuamente, y basta responder en cada disyuntiva a una pregunta para optar por uno u otro camino, facilitando mucho el llegar a un diagnóstico final (si bien adolece de falta de flexibilidad y sería útil principalmente a modo de screening). En medicina humana su uso se encuentra bastante más generalizado que en veterinaria (Yusta, Mateos, \& Rodríguez, 2009; Bates, 2019, p. JC47) y, especialmente, en el campo de la sanidad animal.

Los objetivos de la experiencia fueron: 1) Determinar la utilidad de los algoritmos para el diagnóstico diferencial de los procesos respiratorios y digestivos del cerdo; 2) Establecer el conocimiento por parte del alumnado de esta herramienta diagnóstica y 3) Conocer en qué medida considera el alumnado útil esta práctica de aproximación a la realidad del diagnóstico en el mundo laboral.

\section{DESARROLLO DE LA EXPERIENCIA DE INNOVACIÓN}

El proyecto se estructuró en varias fases; en la primera de las mismas se diseñaron los algoritmos, en la segunda se diseñó la práctica de casos clínicos, incluyendo una última parte de uso de algoritmos para asistir en el diagnóstico y una pequeña encuesta, y una tercera y última parte de análisis de los datos recabados.

\subsection{Diseño de los algoritmos}

Previamente a la fase docente se recopiló información de distintas fuentes para el diseño de los algoritmos, poniendo especial énfasis en los diagnósticos diferenciales de los distintos procesos. Para ello, se buscaron aquellos componentes 
Carbonero, A., Sánchez-Céspedes, R., García-Bocanegra, I., Arenas, A., Luque, I., Tarradas, C., Cano, D. \& Borge, $C$.

epidemiológicos y clínicos específicos de las determinadas enfermedades, es decir, aquellos que permitían dirigirse a un proceso con una respuesta afirmativa y que a su vez englobaban a todo el resto ante una respuesta negativa. De este modo, paso a paso, se crearon los algoritmos que pueden apreciarse en las figuras 1 y 2 .

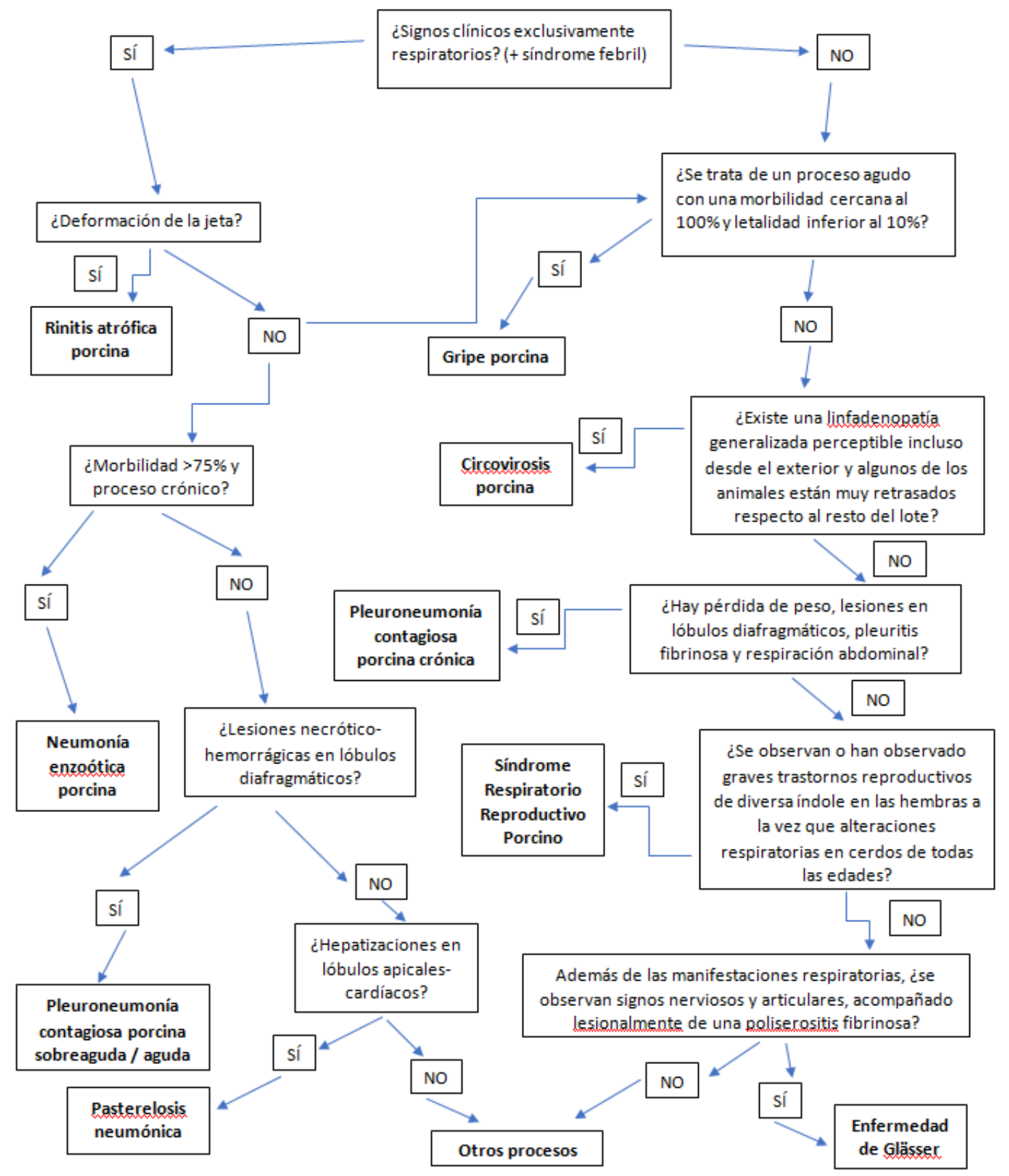

Figura 1. Algoritmo creado para el diagnóstico diferencial de procesos respiratorios en el cerdo. Fuente: Elaboración propia. 


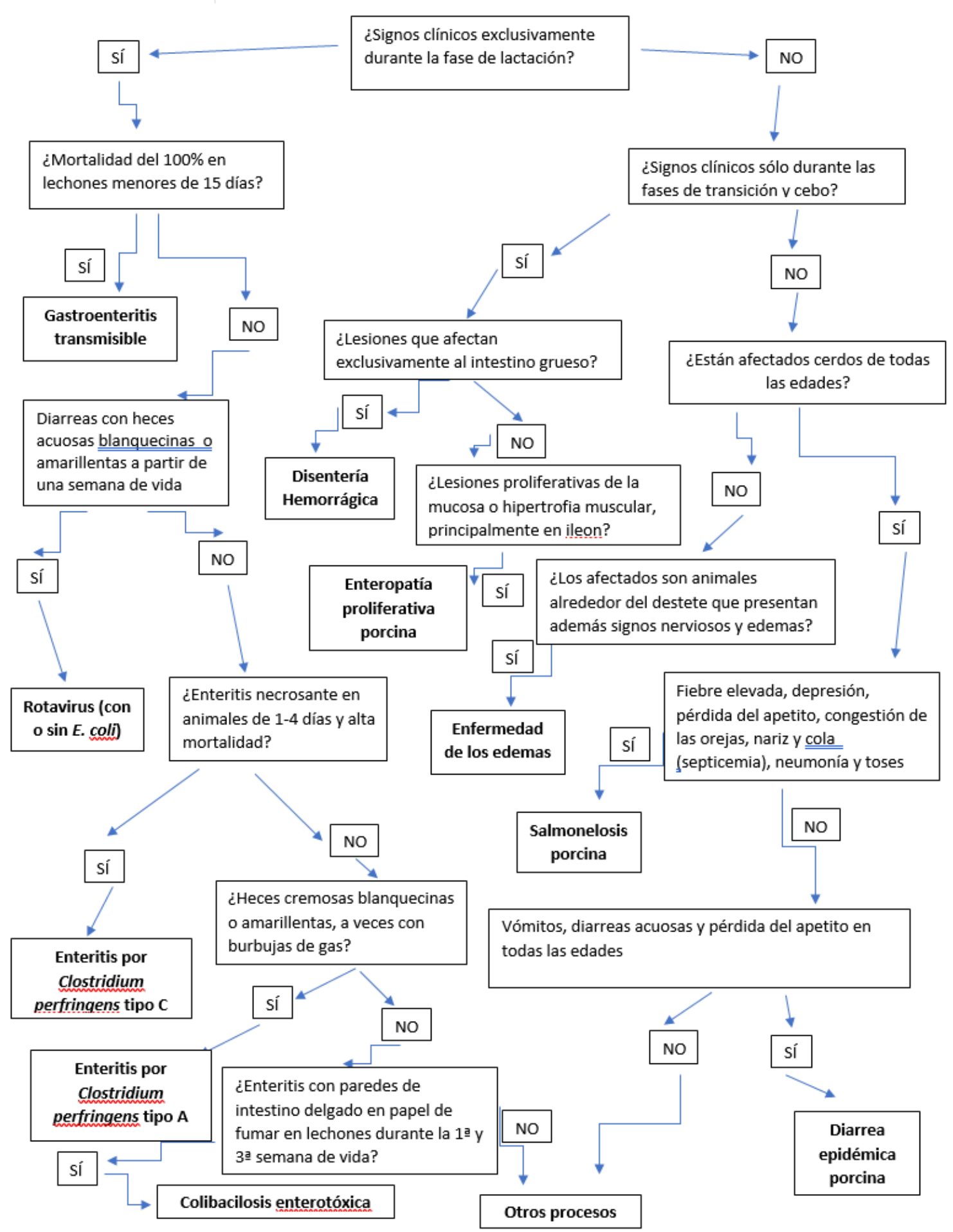

Figura 2. Algoritmo creado para el diagnóstico diferencial de procesos digestivos en el cerdo. Fuente: Elaboración propia. 
Carbonero, A., Sánchez-Céspedes, R., García-Bocanegra, I., Arenas, A., Luque, I., Tarradas, C., Cano, D. \& Borge, $C$.

\subsection{Contexto}

Durante el curso académico 2017-2018 se realizaron tres sesiones prácticas con alumnos matriculados en la asignatura de Enfermedades Infecciosas, asignatura de tercer curso del Grado en Veterinaria en Córdoba (España). En la práctica, cuyo objetivo principal es que los alumnos aprendan a enfrentarse y resolver casos clínicos, se empleó la metodología de estudios dirigidos en grupos pequeños (máximo 12 alumnos).

\subsection{Desarrollo de la práctica}

Tras una concisa explicación de la mecánica de la práctica, los alumnos dispusieron de 10 minutos para completar la anamnesis en un juego de roles donde el profesor actuaba como un ganadero que llamaba por teléfono al verse afectado por un brote en su explotación y los alumnos actuaban como veterinarios con la intención de recabar información. Tras esta fase, el grupo se subdividió en tres grupos de cuatro alumnos, que se separaron en puntos distantes de la clase (figura 3). Estos debían realizar un exhaustivo diagnóstico diferencial y, con objeto de alcanzar un diagnóstico asertivo, solicitar pruebas laboratoriales, con un límite de cuatro. Cuando decidían la prueba a realizar, llamaban al profesor, que actuaba como laboratorio, y especificaban la muestra enviada y el test solicitado, dándole el profesor el resultado. Para reflejar la sensibilidad y especificidad de cada técnica, el profesor tiraba en secreto un dado porcentual (figura 4), dando un resultado de falso positivo o negativo si el resultado de la tirada era superior a la especificidad / sensibilidad, respectivamente.

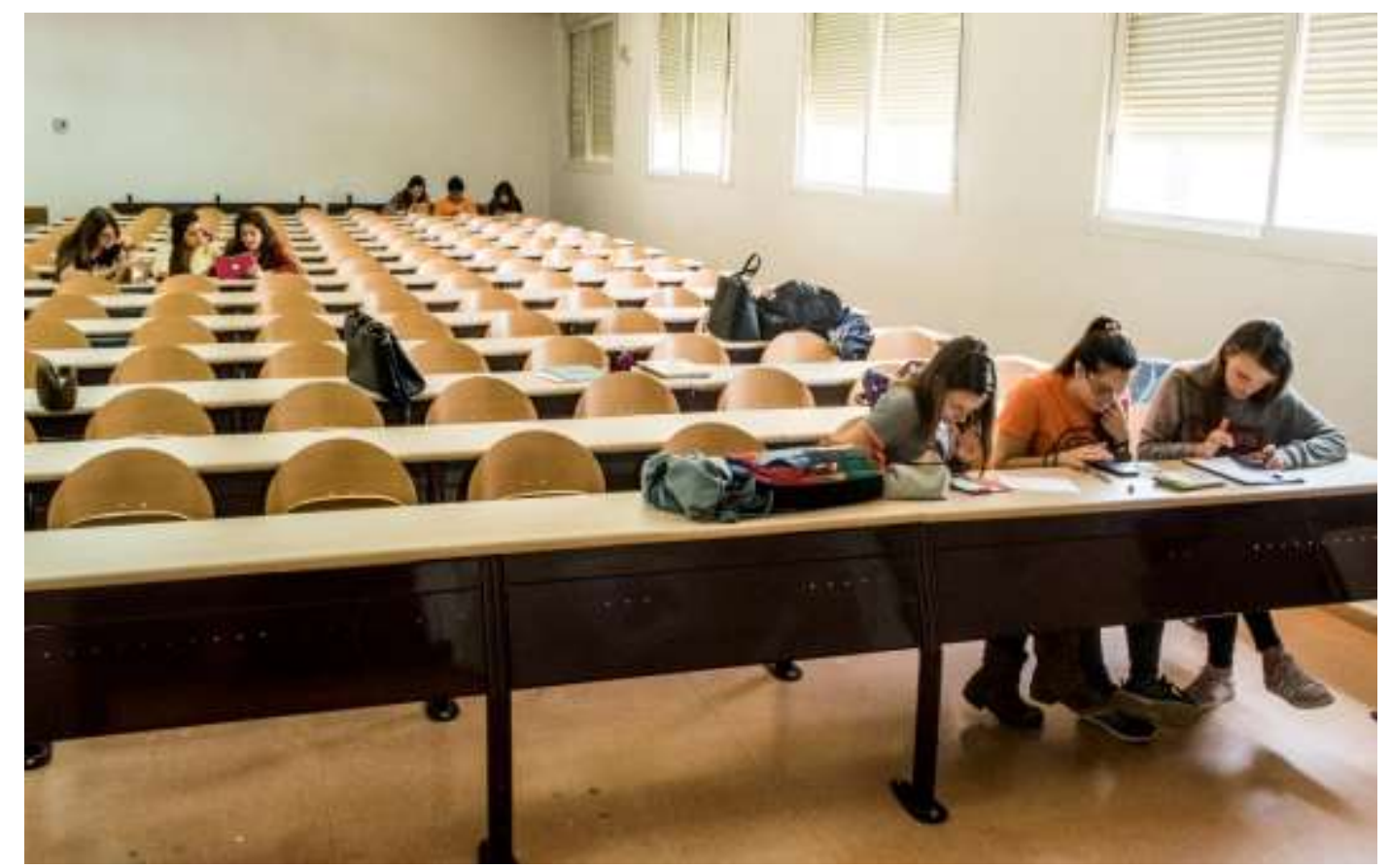

Figura 3. Alumnos separados en subgrupos para la práctica. Fuente: Elaboración propia. 


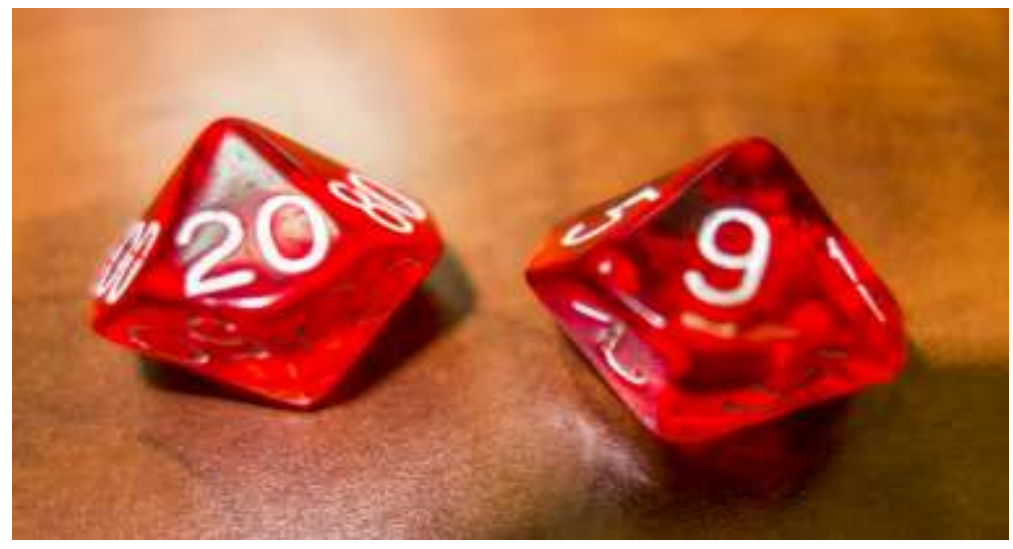

Figura 4. Dados porcentuales empleado para determinar la ocurrencia de falsos positivos/falsos negativos. Fuente: Elaboración propia.

Finalizado el tiempo de que disponían para esta parte (30 minutos), algunos grupos llegaron al diagnóstico correcto y otros no. A continuación, el profesor realizaba una introducción al uso de algoritmos como herramienta de apoyo y les facilitaba los algoritmos creados para las enfermedades respiratorias y digestivas porcinas, dejando cinco minutos para usarlo. Transcurrido este tiempo, se volvían a reunir todos los grupos, se analizaba en conjunto la práctica, enfatizando en la utilidad (o no) de los algoritmos facilitados y, para finalizar, se les pasó una breve encuesta sobre qué les había parecida la práctica en general y el uso de los algoritmos en particular.

\subsection{Análisis de datos}

Para el análisis estadístico de las encuestas se determinaron los parámetros descriptivos de desviación y de tendencia central para las variables cuantitativas, así como la distribución de frecuencias en el caso de las cualitativas. Además, se realizó una prueba $t$ de Student para muestras independientes a fin de evaluar si existían diferencias significativas entre ambos sexos respecto al grado de satisfacción con la práctica, y correlaciones ( $r$ de Pearson) a fin de establecer la existencia o no de asociación del grado de satisfacción con la edad y el curso más alto de matriculación.

\section{RESULTADOS}

En total, se realizaron tres sesiones prácticas en las que participaron 32 estudiantes, lo que supuso 9 subgrupos de diagnóstico. Cuatro de los nueve grupos (44,9\%) diagnosticaron correctamente la enfermedad antes de usar el algoritmo. Una vez se les suministró y lo consultaron, el 100\% de los grupos llegó al diagnóstico correcto. En las tablas 1 y 2 se muestran los resultados del análisis descriptivo de las variables incluidas en la encuesta. 
Carbonero, A., Sánchez-Céspedes, R., García-Bocanegra, I., Arenas, A., Luque, I., Tarradas, C., Cano, D. \& Borge, $C$.

Tabla 1.

Análisis descriptivo de las variables cuantitativas.

\begin{tabular}{|c|c|c|c|c|c|c|}
\hline Variable & Media & Mediana & Moda & $\begin{array}{l}\text { Desviación } \\
\text { típica }\end{array}$ & Mínimo & Máximo \\
\hline Edad (años) & 22,8 & 21,5 & 21 & 3,78 & 20 & 40 \\
\hline $\begin{array}{l}\text { Curso más alto en } \\
\text { que está matriculado }\end{array}$ & 3,75 & 3 & 3 & 0,88 & 3 & 5 \\
\hline $\begin{array}{l}\text { Grado de } \\
\text { satisfacción con la } \\
\text { práctica }(1-10)\end{array}$ & 8,72 & 9 & 9 & 1,02 & 7 & 10 \\
\hline
\end{tabular}

Tabla 2.

Análisis descriptivo de las variables cualitativas.

\begin{tabular}{lcc}
\hline \multicolumn{1}{c}{ Variable } & Categorías & Número (Porcentaje) \\
\hline Sexo & Hombre & $15(46,9)$ \\
& Mujer & $17(53,1)$ \\
\hline Conocimiento previo de la existencia de los & Sí & $16(50)$ \\
algoritmos para el diagnóstico & No & $16(50)$ \\
\hline Utilidad de los algoritmos en la práctica & Sí & $32(100)$ \\
docente & No & $0(0)$ \\
\hline Utilidad de los algoritmos en el ámbito & Sí & $31(96,9)$ \\
profesional & No & $1(3,1)$
\end{tabular}

Fuente: Elaboración propia.

Respecto al grado de satisfacción, se encontraron diferencias a un nivel de significación del $90 \%(p=0,099)$, siendo mayor la satisfacción en las estudiantes de género femenino. Sin embargo, la satisfacción no se correlacionó significativamente ni con la edad $(p=0,819)$ ni con el curso más alto de matriculación $(p=0,352)$.

Los resultados obtenidos permiten establecer el éxito de la actividad práctica diseñada desde una doble perspectiva. Por un lado, se demuestra la utilidad del empleo del algoritmo diseñado, ya que el éxito en el diagnóstico se vio incrementado desde un $44,9 \%$ hasta un $100 \%$. El hecho de que la mitad de los estudiantes desconocieran la utilidad de los algoritmos para el diagnóstico justifica de forma adicional el haberlos incluido como herramienta de apoyo en las sesiones prácticas. Por otra parte, los estudiantes han manifestado un notable grado de satisfacción con la práctica, con una media de 8,72 puntos sobre 10.

\section{CONCLUSIONES}

La práctica ha resultado, tanto en opinión de los alumnos en la del profesorado participante, un auténtico éxito. Los algoritmos, desconocidos para la mitad de los estudiantes participantes, se han revelado como una importante herramienta para alcanzar un diagnóstico correcto, ordenando los conocimientos de una forma sistematizada. Sin embargo, habría sido conveniente el haber probado este tipo de práctica en un mayor número de grupos para dar una mayor posibilidad a la obtención de diferencias significativas. Como mejora, se podría ampliar en proyectos futuros el empleo de los algoritmos a otro tipo de sintomatologías (nerviosas, reproductivas, etcétera) y a otras especies además del cerdo. 


\section{REFERENCIAS}

Bates, E. R. (2019). In patients with suspected AMI and LBBB, algorithms based on ECG and troponin data were tested to rule MI in or out. Annals of Internal Medicine, 171, JC47.

Chandramohan, D., Jaffar, S. \& Greenwood, B. (2002). Use of clinical algorithms for diagnosing malaria. Tropical Animal Health and Production, 7, 45-52.

Fueyo, Di Domenicantonio, R., Cappai, G., Agabiti, N., Marino, C., Simonato, L., Canova, C. \& Pitter, G. (2019). A Systematic Review of Case-Identification Algorithms Based on Italian Healthcare Administrative Databases for Three Relevant Diseases of the Digestive and Genitourinary System: Inflammatory Bowel Diseases, Celiac Disease, and Chronic Kidney Disease. Epidemiologia e prevenzione, 43, 88-98.

Martin, C. M., Doig, G. S., Heyland, D. K., Morrison, T. \& Sibbald, W. J. 2004. Multicentre, cluster-randomized clinical trial of algorithms for critical-care enteral and parenteral therapy. Canadian Medical Association Journal, 170, 197-204.

Yusta, A., Mateos, J. \& Rodríguez, M. (eds). (2009). Algoritmos Clínicos en Medicina, (2 $2^{-}$ed.). Madrid, España: Grünenthal, S.A. 